\title{
MODELLING AND SIMULATION OF IMPEDANCE-BASED DAMAGE MONITORING OF STRUCTURES
}

\author{
Djemana, M. \& Hrairi, M. \\ Department of Mechanical Engineering, International Islamic University Malaysia, P. O. Box 10, \\ 50728 Kuala Lumpur, Malaysia \\ E-Mail: dj23mohamed@gmail.com, meftah@iium.edu.my ( ${ }^{*}$ Corresponding author)
}

\begin{abstract}
Electromechanical impedance (EMI) based monitoring techniques are successfully in use in current engineering structures. With the help of piezoelectric sensors, the EMI technique is used for monitoring the health of such structures. Generally, potential damage to the host structure is detected by examining the EMI signature and identifying changes in that unique signature. Since this technique has the potential to offer greater safety and reliability while lowering maintenance costs, it is becoming increasingly popular. This paper investigates the use of finite element method (FEM) to simulate the electro-mechanical impedance technique. A numerical analysis of simple models, such as free piezoelectric patches of various shapes and thicknesses is used to comprehend the fundamentals of this technique. Then, studies on different parts of the structure are conducted to find the effect on the output of system when both damage and loading co-exist, and investigate the effect of temperature for structural health monitoring based on EMI. The simulation results are then compared to experimental data and a very good agreement is observed.

(Received in July 2015, accepted in March 2016. This paper was with the authors 3 months for 2 revisions.)
\end{abstract}

Key Words: Structural Health Monitoring, Electromechanical Impedance, Piezoelectric Patch, Finite Element, Simulation

\section{INTRODUCTION}

There is a wide array of non-destructive evaluation (NDE) techniques used for damage detection including eddy currents, magnetic field analysis, ultrasonic wave propagation, dye penetrant test, acoustic emission, and x-ray radiography. However, all of these techniques tend to be dependent on visual inspection to locate the potential damage before evaluation. Depending on human visual inspection of a large structure can be both tedious and inefficient. For example, some critical parts of a large structure may be hidden or inaccessible. This situation is compounded by some inspection techniques' requirements of bulky equipment and probes, as well as potential exposure to dangerous conditions or uncomfortable working environments. Visual inspection is neither economical nor reliable because it depends on the inspector's experience and potentially unsystematic procedures [1].

Recently, there is a push to achieve structural health monitoring (SHM) using techniques based on smart materials, such as piezoelectric (PZT) materials, rather than the conventional NDE techniques. Compared to conventional techniques, smart materials cost less and are more reliable as they work autonomously in real-time. Electromechanical impedance (EMI) is a smart material technique that employs PZT transducers with a reputation for sensitivity required to detect local damage. Additionally, the EMI technique has been continuously improved over recent decades due to its attractive attributes such as being cost-effective, extremely light, noninvasive, and able to perform self-actuating and sensing.

Hamzeloo et al. [2] detected damage on hollow cylinders using the EMI method. Accumulated damage was shown to affect the damage metrics obtained both experimentally and by finite element method (FEM). Results from the simulation and experiments showed that the damage metrics were affected by the longitudinal distance from the sensor location and its orientation. Moslemi and Khoshravan [3] proposed a methodology for the 
delamination characterization of composite laminates under pure Mode I. Different methods of determining the damage index were presented by Azhdary and Shabakhty [4]. The obtained results showed that the geometric characteristics of structural elements have a great influence on the sensitivity of damage index of structures [4].

A method to locate damage and create a damage map, based on both data indicators computed from electromechanical impedance spectrums and inverse distance weighing interpolation had been proposed by Cherrier et al. [5]. The electromechanical impedance principle was the basis for a new method of damage detection in structures presented by Cortez et al. [6]. The system did not compute the impedance but rather followed variations in piezoelectric transducer's voltage output. An experimental study by Baptista et al. [7] focused on the temperature effects on the electrical impedance of the piezoelectric sensors used in the EMI technique. The experimental results indicated a strong correlation between the effects of temperature and frequency-dependence. The results suggest future research in the SHM field may be required.

Furthermore, PZT transducers can monitor damage when loading is absent and can be equally efficient at monitoring loading when damage is absent [8]. However, efficiency of EMI when both damage and loading are present in the structure requires additional research. Another issue facing EMI is detecting damage while there are temperature effects, because it is an especially critical problem when low damage levels are to be detected. There is still a need to develop efficient temperature compensation methods for EMI SHM. In SHM application, the most important damage indicator is the change in the admittance signature of the bonded PZT transducer. Most of the research that has been done in this area explored either analytical or experimental solutions. In many cases, doing experiments is very expensive and also very difficult, if not impossible. In this paper, numerical analyses and simulations using FEM will be performed to understand the concept of the technique. This simulation approach will be carried out on different types of structural members to detect the locations of damage and investigate the effect of temperature. In particular, the focus will be on bridging two research gaps. On one hand, to investigate the efficiency of EMI when both damage and loading are present in the structure. On the other hand, to develop efficient temperature compensation method so that EMI can detect low damage levels while there are temperature effects.

\section{IMPEDANCE-BASED STRUCTURAL HEALTH MONITORING}

Piezoelectric transducers have evolved as an efficient smart material that can react to highfrequency structural excitations in the host structure to create a unique health signature [9]. The method for obtaining these signatures, as an inverse function of structural impedance, is termed as the electromechanical impedance technique [8]. The base concept underlying the EMI technique is associated with the change of electrical impedance of PZT patches by the following equation:

$$
z(\omega)=\left[i \omega C\left(1-k_{31}^{2} \frac{Z_{s t r}(\omega)}{Z_{P Z T}(\omega)+Z_{s t r}(\omega)}\right)\right]^{-1}
$$

where $Z(\omega)$ is the equivalent electromechanical impedance, as seen at the PZT transducer terminals, $C$ is the zero-load capacitance of the PZT transducer, $k_{31}$ is the electro-mechanical cross coupling coefficient of the PZT transducer $k_{31}=d_{31} / S_{11} \times \varepsilon_{33}$, where $d_{31}$ is the piezoelectric constant; $S_{11}$ is the compliance, $\varepsilon_{33}$ is the dielectric permittivity, $Z_{s t r}$ is the mechanical impedance of the structure, and $Z_{P Z T}$ is the mechanical impedance of the PZT transducer [10]. 
The piezoelectric sensor-actuators are closely bonded to the structure, and their EMI variation is measured across a wide frequency spectrum located in the high $\mathrm{kHz}$ band of frequencies. The transducer frequency response, phase, and amplitude, the impedance response (both real and imaginary parts), and other significant physical parameters act as indicators of potential structure damage and reflect the level of structural integrity. The transducer approach has proven most effective in the ultrasonic range of frequencies, where changes in local dynamics due to the initiation of damage within the structure are captured. Changes at this nascent stage are very small and have minimal effect on the global dynamics of the structure, making them difficult to detect with traditional low frequency vibration techniques [11].

\section{PIEZOELECTRIC ON HEALTHY ALUMINIUM BEAM}

\subsection{Free piezoelectric patch model}

At first, a free PZT patch was modelled, using ANSYS multi-physics software, in order to understand the fundamentals of the FE modelling approach and its efficiency. A free square shaped patch of $7 \times 7 \times 0.2 \mathrm{~mm}^{3}$ in size, made of APC-850, was modelled. The coupled-field finite element SOLID 226, that has 20 nodes with up to six degrees of freedom $(D O F)$ at each node, was used. For piezoelectric analysis, an additional $D O F$, the electric voltage can be added to the displacement $D O F$ s. The electrical charge will be used to calculate the electromechanical impedance of the system. The admittance of the system $(Y)$ can be calculated using the output charge $(q)$ and the applied voltage $(V)$ to the PZT patch using the following formula:

$$
Y=\frac{I}{V}
$$

where the electrical current $(I)$ is expressed as a function of the radial frequency $(\omega)$ and the complex charge $(q)$ :

$$
I=i \omega q
$$

and $\omega$ is expressed as a function of the system frequency $(f)$ :

$$
\omega=2 \pi f
$$

Using these formulas, the admittance is finally obtained by Eq. (5) and the electromechanical impedance by Eq. (6):

$$
\begin{gathered}
Y=i 2 \pi f q / V \\
Z=1 / Y
\end{gathered}
$$

The simulation was done using $400 \mathrm{sub}$ steps from 0 to $1000 \mathrm{kHz}$ for a freely suspended square patch. Fig. 1 shows the FE model of the free PZT patch and the comparison between the simulation and the experimental results presented in Gresil et al. [12]. The real part of the impedance was used in preference over the imaginary part. This selection was made because the real part is known to have more reaction to damage or changes in the structure's integrity [13]. Generally, good matching is observed as the signature of the impedance is displaying similar resonant frequencies but some differences are visible. For frequencies above $800 \mathrm{kHz}$, there is a peak in the simulation which is not available in the experimental result. This could be related to the number of sub steps between the starting and ending frequency, taking as 400 in the simulation but was not mentioned in the experimental work. Furthermore, small differences are expected in the numerical response when compared to the experimental 
impedance because the wiring was not modelled and unavoidable and measuring errors may also occur. Nonetheless, this comparison illustrates that reasonable agreement can be obtained when FEM method is used, which is an important improvement over the use of the analytical models [12]. The noticeable difference is only observed in terms of the upward shifting of the experimental results due to the usage of a resistor. The latter was used in the measuring systems of the previous studies $[12,14]$. The variation due to this difference is just in terms of anti-resonance. As shown in Fig. 2, the effect of introducing the resistor to the system was only in upward shifting not the changing in resonant frequency or even the overall trend. Hence, it can be concluded that it is safe to not consider modelling the resistor for all simulation cases as only the upward shifting is expected when a resistor is involved.
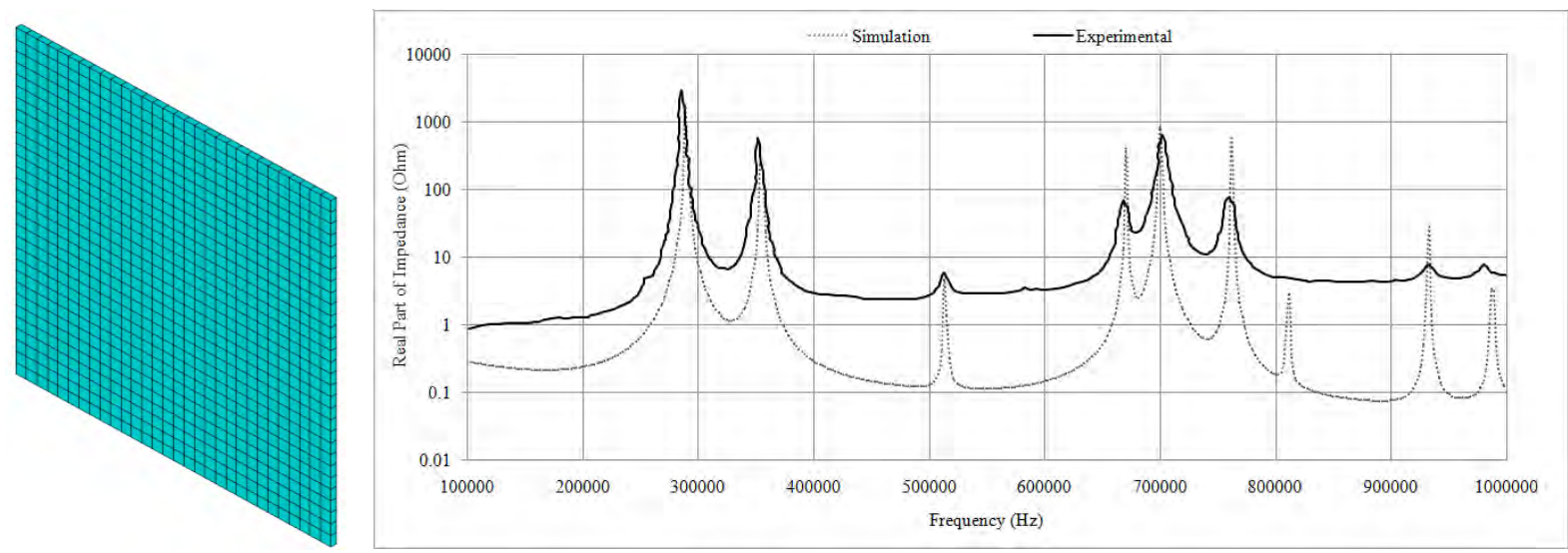

Figure 1: Free square PZT patch model (left) and numerical and experimental results of impedance real part (right).
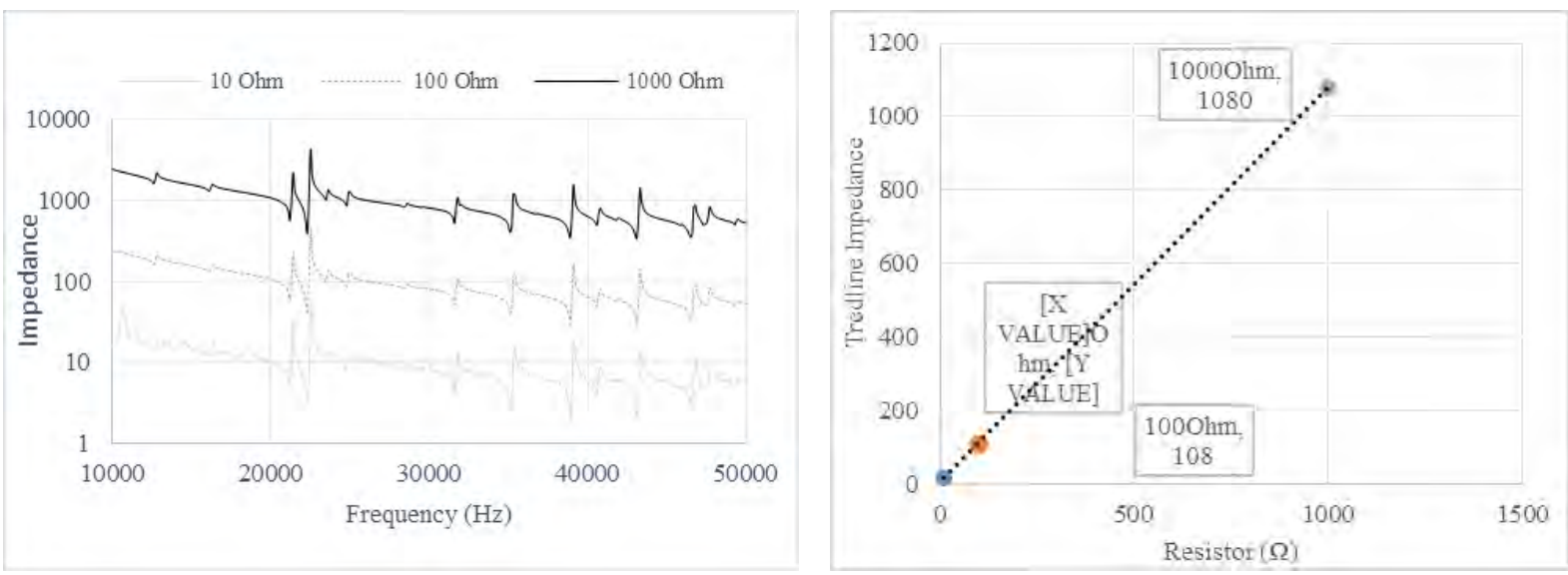

Figure 2: Effect of resistor on magnitude of impedance.

\subsection{Piezoelectric patch bonded to a beam model}

In order to implement the mechanical coupling between the sensor and the structure, the sensor boundary conditions are specified while multi-physics equations are used to model the piezoelectric material for the electromechanical coupling. The first coupling transforms the mechanical response sensed by the piezoelectric element into an electric signature. The aluminium alloy 1100 structure was $100 \mathrm{~mm} \times 16 \mathrm{~mm} \times 1 \mathrm{~mm}$ while the PIC151 PZT patch had the dimensions of $10 \mathrm{~mm} \times 10 \mathrm{~mm} \times 0.3 \mathrm{~mm}$ and was located $5 \mathrm{~mm}$ from the beam's left edge. The model also considered a thin layer of epoxy adhesive with $0.03 \mathrm{~mm}$ thickness used to bond the sensor to the structure. The mechanical properties of the materials are detailed in Tables I and II. 
Table I: Material properties of beam and epoxy adhesive.

\begin{tabular}{|c|c|c|c|c|}
\hline Parameters & Symbols & Materials & Values & Unit \\
\hline Density & $\rho$ & $\begin{array}{l}\text { Aluminium } \\
\text { Epoxy }\end{array}$ & $\begin{array}{l}2.715 \\
1.000\end{array}$ & {$\left[\mathrm{~kg} / \mathrm{m}^{3}\right]$} \\
\hline Poisson ratio & $V$ & $\begin{array}{l}\text { Aluminium } \\
\text { Epoxy }\end{array}$ & $\begin{array}{l}0.3 \\
0.4 \\
\end{array}$ & - \\
\hline $\begin{array}{l}\text { Young's modulus } \\
\text { (Isotropic) }\end{array}$ & $E$ & $\begin{array}{l}\text { Aluminium } \\
\text { Epoxy }\end{array}$ & $\begin{array}{c}68.95 \\
5.1\end{array}$ & $10^{9}\left[\mathrm{~N} / \mathrm{m}^{2}\right]$ \\
\hline
\end{tabular}

Table II: Material properties of PI151.

\begin{tabular}{|c|c|c|c|}
\hline Parameters & Symbols & Values & Unit \\
\hline Density & $\rho$ & 7800 & {$\left[\mathrm{~kg} / \mathrm{m}^{3}\right]$} \\
\hline Compliance & $S_{11}=S_{22}$ & 19.0 & - \\
\hline \multirow{2}{*}{ Electric permittivity coefficient } & $\varepsilon_{11}=\varepsilon_{22}$ & 1977 & - \\
& $\varepsilon_{33}$ & 2395 & - \\
\hline \multirow{2}{*}{ Piezoelectric strain coefficient } & $d_{31}=d_{32}$ & -2.10 & {$\left[10^{-10} \mathrm{~m} / \mathrm{V}\right]$ or } \\
& $d_{33}$ & 5.00 & {$\left[10^{-10} \mathrm{C} / \mathrm{N}\right]$} \\
\hline
\end{tabular}

The elements solid226 and solid95 were employed to model the PZT patch and the metallic host structure respectively. Frequency plots of electromechanical impedance were generated with the help of harmonic analysis performed with the elaborated finite element model. Voltage levels of $1 \mathrm{~V}$ and $0 \mathrm{~V}$ (ground) were applied to the top and bottom master nodes respectively. Fig. 3 shows the FE model of PZT patch bonded on a healthy beam and Fig. 4 illustrates the comparison of the real portion of the impedance simulation results with the experimental ones obtained by Rosiek et al. [14]. Most of the peaks in the comparison were located at the same frequencies; however there was a shift to the left noticeable for some peaks. These shifts may have been caused by the absence of values for the elastic material's properties that were used in the experiment. In addition, the difference between the simulated and the measured value remains in particular frequency domains. This difference between the $0.03 \mathrm{~mm}$ thick adhesive layer and the simulation can be possibly explained by an uneven or degraded bond-line between the sensor and the host structure. This would also be consistent with the fact that, for thinner adhesive layer, the experimental data [15] showed a decrease in the peak frequency compared with the thicker cases, suggesting that a weaker bond between the sensor and the host structure may be present. Furthermore, at higher frequencies, the adhesive thickness-shear modulus ratio has significant influence on the resonance amplitudes and frequencies [16].

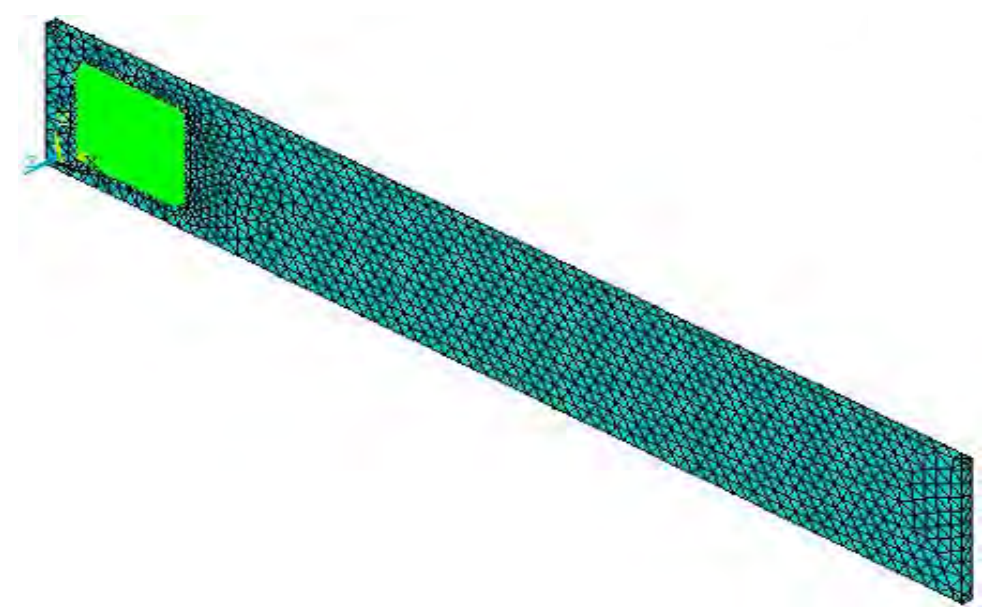

Figure 3: FE model for square patch on healthy beam. 


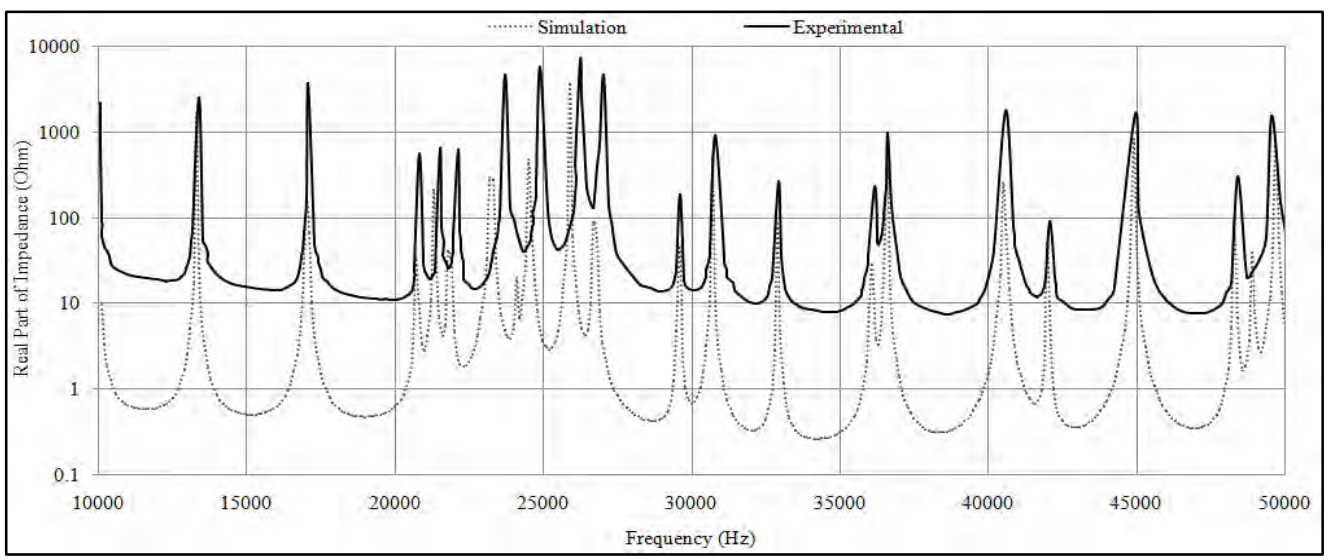

Figure 4: Impedance response of a bonded square patch on healthy beam

\subsection{Effect of meshing}

To ensure sufficient accuracy of the solution, proper element size had to be chosen. Three different simulations using element sizes of $0.2 \mathrm{~mm}, 0.5 \mathrm{~mm}$ and $1 \mathrm{~mm}$ were carried out to see the effect of meshing on impedance. As shown in Fig. 5, different mesh sizes did not affect the trend very much, thus it is acceptable to use the mesh size of $0.5 \mathrm{~mm}$, as it does not have too many nodes and new peaks exist, compared to $1.0 \mathrm{~mm}$ mesh size. More peaks make it easier to spot the changing in impedance behaviour for future cases.

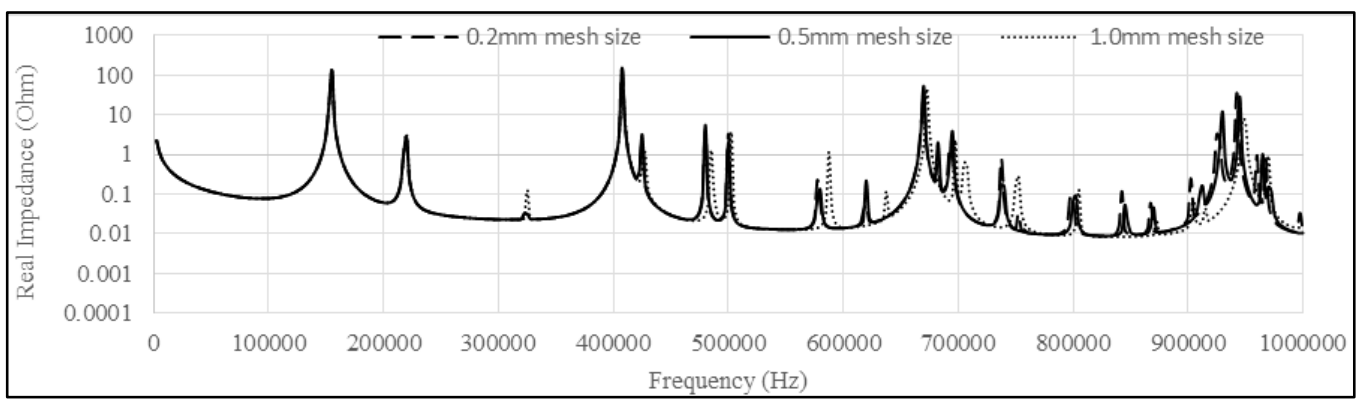

Figure 5: Effect of mesh sizes on the real portion of impedance.

\subsection{Effect of patch shape}

Different shapes of PZT patches were modelled in order to study the effect of shape on the impedance signature. In this case, square, circular and rectangular shapes having the same area and thickness were modelled. Fig. 6 depicts the difference in impedance real part signatures when using the two distinctive patch shapes. It can be seen that, unlike the rectangular patch, both square and circular patches behave in a relatively similar manner. The slight difference occurs because of the number of elements used to mesh the patch shape. The circular patch needed to use sweep hexagonal elements, due to the curvature geometric characteristic, whereas the square patch required mapped hexagonal elements. These parameters affect the number of elements generated. Consequently, the circular patch contained a higher number of elements, hence the slight difference in its impedance plot compared to the one obtained by the square patch. Furthermore, the circular shape has the lowest number of peaks followed by circular and then rectangular patch. These differences can be related to the symmetrical shape of the patch. As long as the patch has a better symmetrical parameter, the number of peaks is less. It is also worth noting that choosing between these patches for the EMI technique is related to the shape of the host structure and frequency range. For example, for lower frequencies, it is better to use the rectangle shape but 
for higher frequencies, using these patches will complicate the result by giving a lot of peaks. In the EMI technique, the circular and square patches are used more than other shapes.

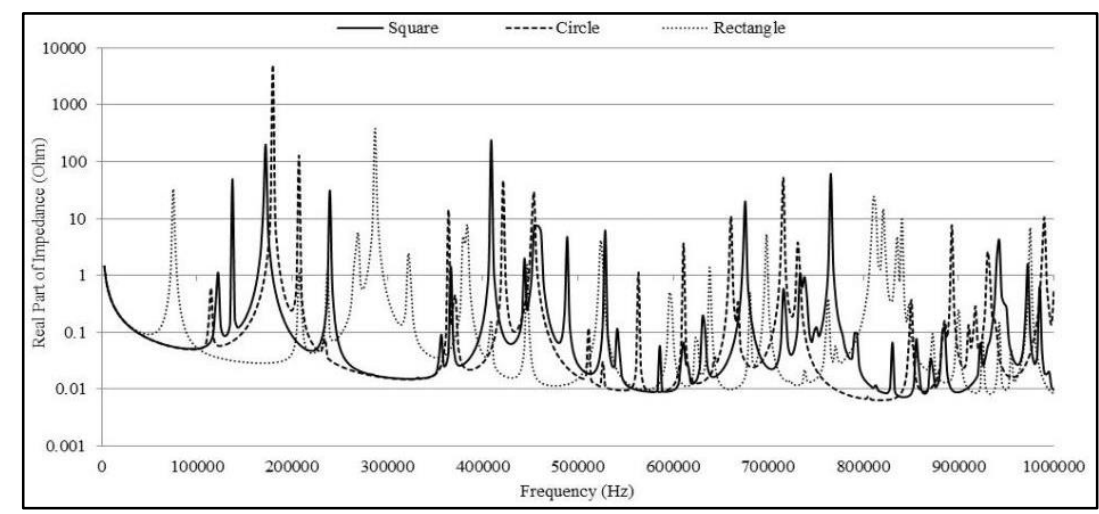

Figure 6: Comparison of impedance when square, circular or rectangular patch is bonded to the beam.

\subsection{Effect of patch thickness}

For this case, a PZT patch bonded to the aluminium beam structure with different thicknesses was modelled at a position of $5 \mathrm{~mm}$ distance from the beam's left edge (Fig. 3) to discover how PZT patch thickness affects the EMI technique. The thicknesses were 0.05, 0.3 and 1 $\mathrm{mm}$. Based on Fig. 7, only the amplitudes increase when the patch thickness increases. Thicker patches affect the global stiffness, and when it is bonded to the beam, the stiffness of the system rises. According to the results obtained by Baptista et al. [17], the patch must be small to ensure low static capacitance and high amplitude in the electrical impedance. PZT patches of sizes ranging from 5 to $15 \mathrm{~mm}$ and thicknesses of 0.1 to $0.3 \mathrm{~mm}$ are best suited for most structural materials such as metals. Such thin patches usually have a thickness resonance frequency in the order of a few MHz. Therefore, the frequency response signature in the $\mathrm{kHz}$ frequency range is characteristic of the structure only [18]. However, for thicker patch $(1 \mathrm{~mm}$ in Fig. 7), the thickness resonance frequency will get lower and the frequency response signature in the $\mathrm{kHz}$ frequency is slightly affected by the patch especially at higher frequencies above $600 \mathrm{kHz}$. Therefore, a thinner patch of $0.3 \mathrm{~mm}$ thickness was used in this research, in order to allow results comparison with data from the literature [18] that used the same patch dimension.

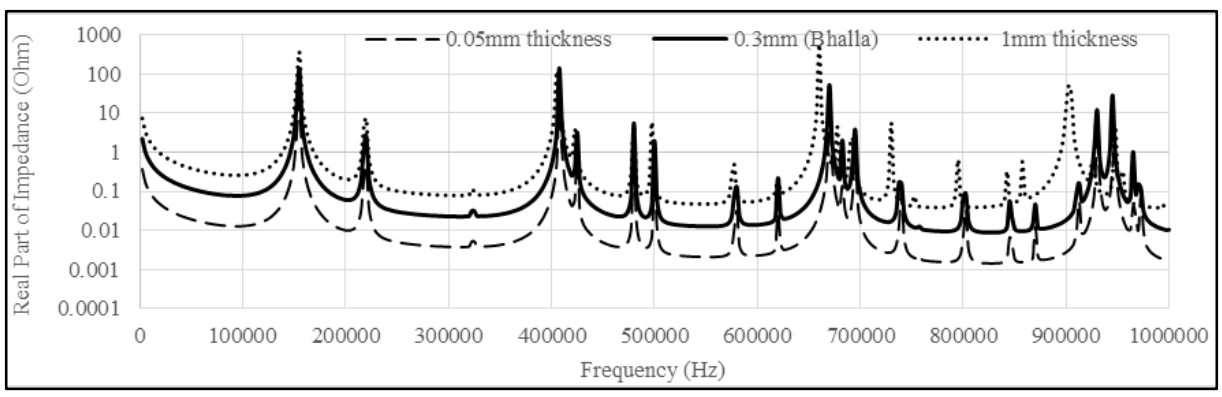

Figure 7: Effect of patch thickness on real part of impedance.

\subsection{Effect of glue thickness}

The adhesive layer is another important parameter that has significant effect on the EMI system. This layer transfers the signals bi-directionally between the PZT patch and the structure. The properties and quality of this layer are very important. Fig. 8 depicts the link between bonding layer thickness and impedance response. As illustrated in Fig. 8, high repeatability of the impedance signatures could be observed for patches bonded on the beam 
specimen but with different bonding thicknesses. In other words, they possess identical PZTstructure interaction even with different bonding thicknesses. All resonance peaks could be accurately matched, indicating that the PZT patches were consistent in the process of sensing, despite the minor variations in magnitude caused by different bonding thicknesses. It is apparent that as the bond layer thickness increases, the peaks subside down and shift rightward. This effect has also been observed by Bhalla et al. [18] and Ong et al. [19]. Therefore, the recommendation given by Bhalla [18] stating that the bonding thickness shall not exceed one-third of the PZT patch's thickness was further verified. In this case, thick bonding should be avoided to reduce the contamination in the structural resonance peaks caused by the PZT resonances. Various experimental case studies [20] showed that the effect of bonding can be neglected even for thickness up to two thirds of the PZT patch's thickness, provided that the excitation frequency does not exceed $100 \mathrm{kHz}$. Above this frequency, the adverse effect of thick (larger than 1/3 of PZT thickness) bonding is obvious and thin bonding layer which is less than $1 / 3$ of PZT thickness is recommended. In our case, one-third of the PZT's thickness is $0.1 \mathrm{~mm}$, the bonding layer thickness is $0.03 \mathrm{~mm}$ and the range of frequency is up to $50 \mathrm{kHz}$. Consequently, the thickness of the glue has almost no impact upon the impedance. It only affects some amplitude, but in general, epoxy adhesive thickness will not have much effect in terms of the impedance signature trend.

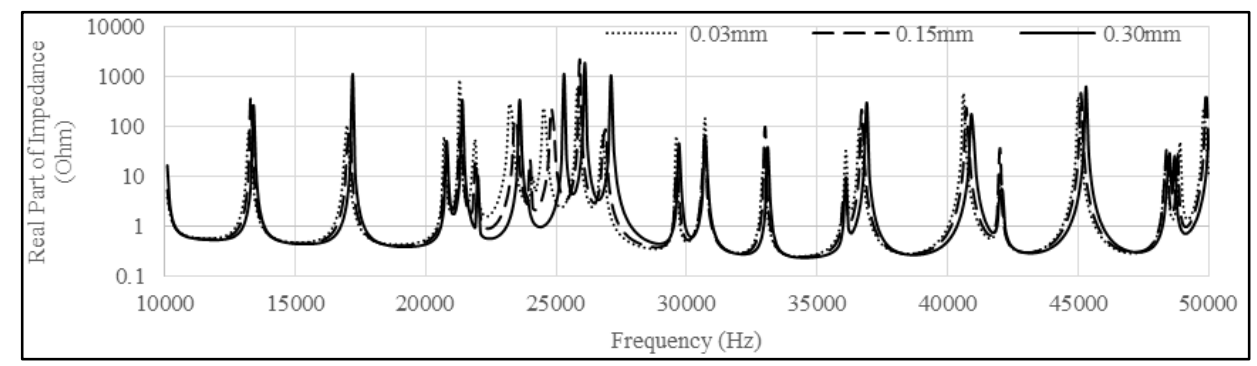

Figure 8: Glue thickness effect on the real part of impedance.

\section{MODELLING OF DAMAGE DETECTION}

\subsection{Effect of notch size}

In this section, structural damage was simulated (Fig. 9). The damage took the form of a $0.001 \mathrm{~m}$ wide incision located $0.055 \mathrm{~m}$ from the beam's left vertical edge. The depth of the incision varied between 0.001 to $0.004 \mathrm{~m}$. Similar analyses have been performed to generate the frequency plots of electromechanical impedance for both healthy construction and with introduced damage. Fig. 10 shows the EMI plot results of the harmonic analysis for both healthy and damaged structures. Since the real component of the EMI is more sensitive to incipient damage, it is the only component used for failure assessment. Fig. 10 shows that by increasing the size of the crack, the peaks are shifted to the left side due to the decreasing stiffness of the structure. For instance, the $0.004 \mathrm{~m}$ damage graph is shifted more than $0.003 \mathrm{~m}$ to the left. Also, by increasing the size of the crack, new peaks appeared because of significant structural changes.

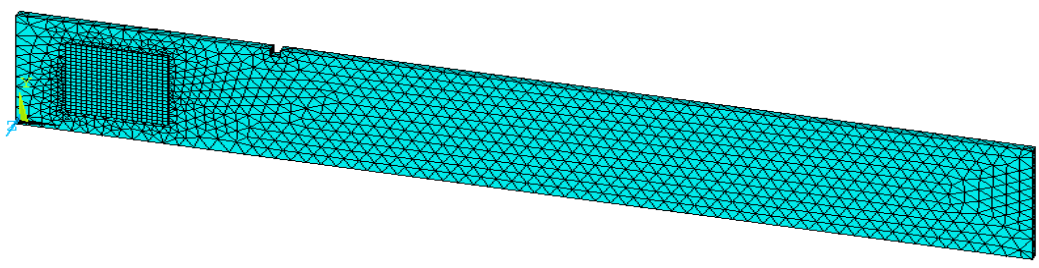

Figure 9: Aluminium beam with a notch crack. 


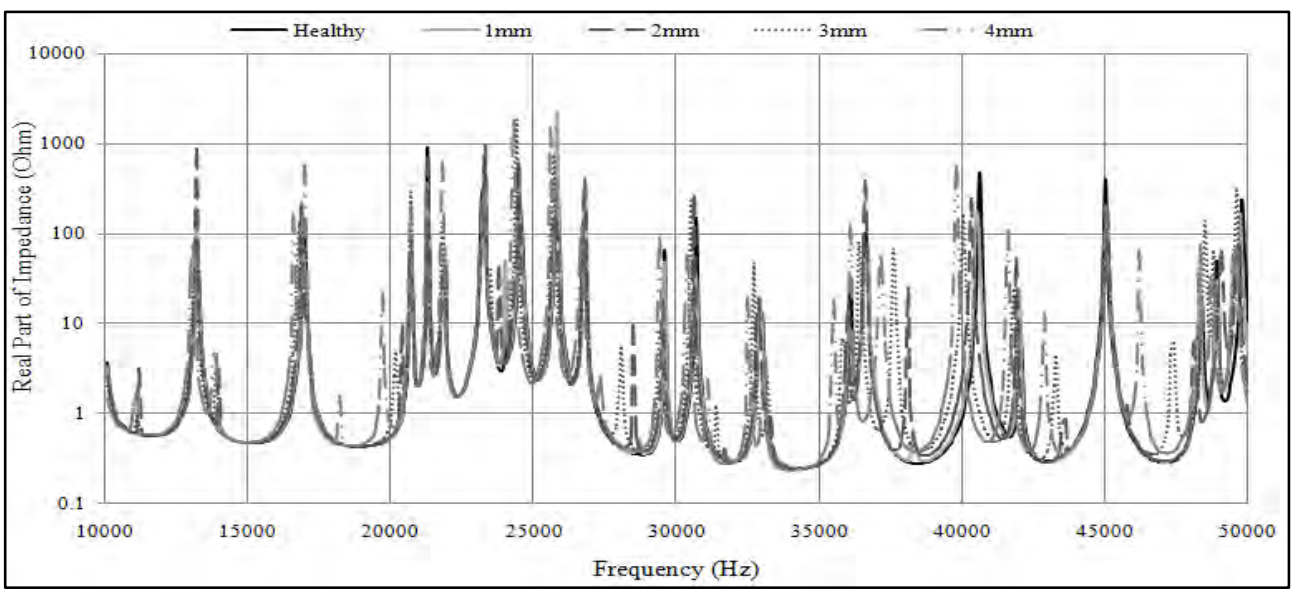

Figure 10: Comparison of impedance between healthy and different notches size.

Fig. 11 shows the electrical impedance signatures (the real part of impedance) that were obtained for the healthy structure (baseline signature) at the resonance frequency of $40.6 \mathrm{kHz}$ and the different damage sizes induced to the structure. The resonance peak clearly exhibited left frequency shifts as the damage increased. .Therefore, the frequency shifts depended on both the length of notches and the frequency. Only a narrow frequency band of $39-41.4 \mathrm{kHz}$ is shown to facilitate the comparison of the impedance signatures.

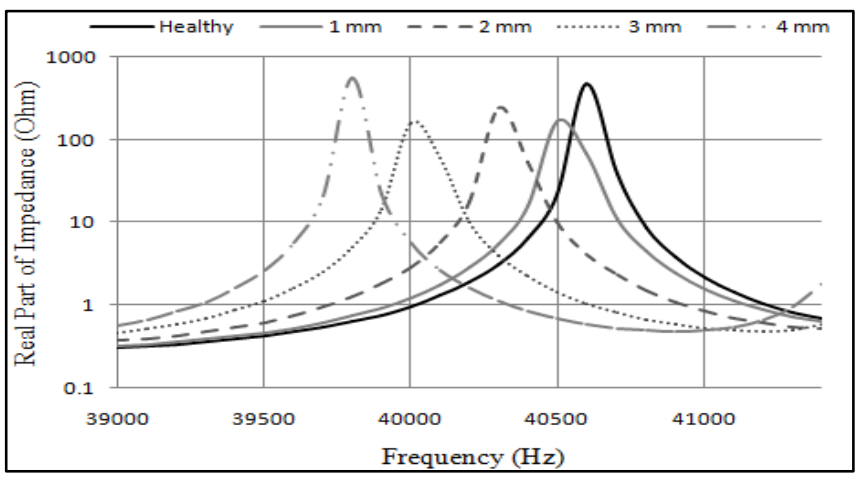

Figure 11: Frequency shifts of the real part of the real part signature for different notch size.

Although EMI signature change can be observed visually, it is necessary to employ statistical techniques to quantify it. Among these techniques, root mean square index (RMSD) is usually applied to characterize structural damages. Mathematically, RMSD is defined as

$$
R M S D=\sum_{K=1}^{\omega_{F}} \sqrt{\frac{\left[R_{E}\left(z_{E, D}(k)\right)-R_{E}\left(z_{E, H}(k)\right)\right]^{2}}{R_{E}^{2}\left(z_{E, H}(k)\right)}}
$$

where the subscripts $D$ and $H$ indicate damaged and healthy conditions, respectively; $R_{E}\left(Z_{E, D}(k)\right)$ and $R_{E}\left(Z_{E, H}(k)\right)$ are the real parts of the electrical impedance signatures after and before damage, respectively. The range of frequency $k$ is measured between the initial and final frequencies.

It is well known, frequencies below $100 \mathrm{kHz}$, particularly in the $0-50 \mathrm{kHz}$ band, produce higher damage indices than other frequency bands [20]. Therefore, in this study, the RMSD index was calculated over a $39-41.4 \mathrm{kHz}$ frequency range. Fig. 12 shows the quantification and identification of damage using the $R M S D$ index of damage conditions $1-4 \mathrm{~mm}^{2}$. As it can be seen, the $R M S D$ is proportionate to the amount of damage incurred. The damage conditions $1 \mathrm{~mm}$ and $2 \mathrm{~mm}$ are small in these results due to the lack of sensitivity in the PZT for the damage signal in the aluminium. However, for the more significant damage depth of $4 \mathrm{~mm}$ 
(one quarter of the beam's full width), the indices show obvious correlation to the damage. The analysis results support the premise that the EMI method can be used to monitor structural damage in aluminium beams. Normalization of the results was performed by setting $R M S D=1$ in the baseline healthy structure.

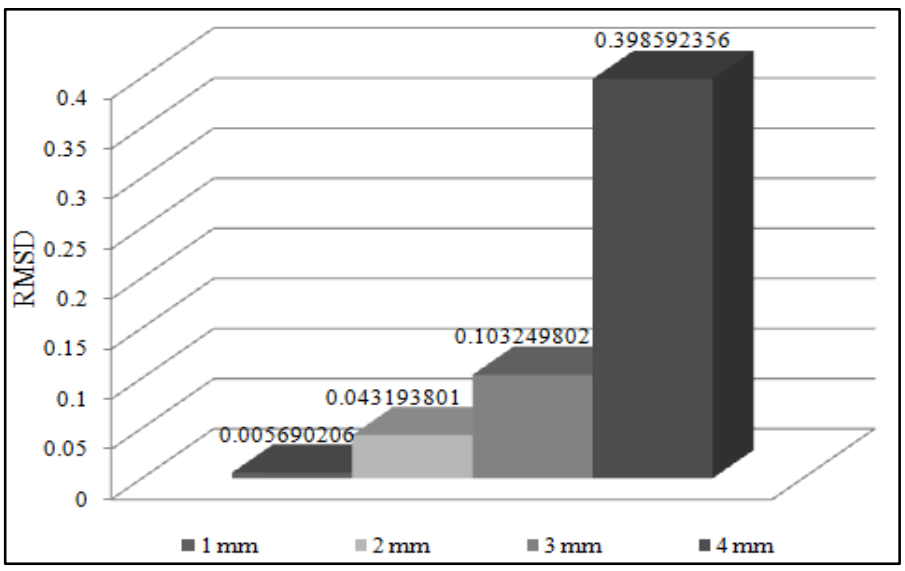

Figure 12: Histogram of the RMSD index of different damage sizes.

\subsection{Effect of patch location from notch}

For this case, the PZT patch was placed at different positions on the beam in order to find the position effect on the output of EMI system. Fig. 13 shows the result for different positions of the PZT having constant damage. The overall trend does not change, but the amplitude seems to be affected. The furthest location tends to yield lower amplitude, whereas the nearest one showed the highest peaks. The distance of $35 \mathrm{~mm}$ and $25 \mathrm{~mm}$ produced fairly similar results, where the highest peaks were interchangeable between the two. The greater distance from the damage contributed to the loss, since the area surrounding the notch produced the highest strain. The impedance values reduced as the length between the patch and the notch increased.

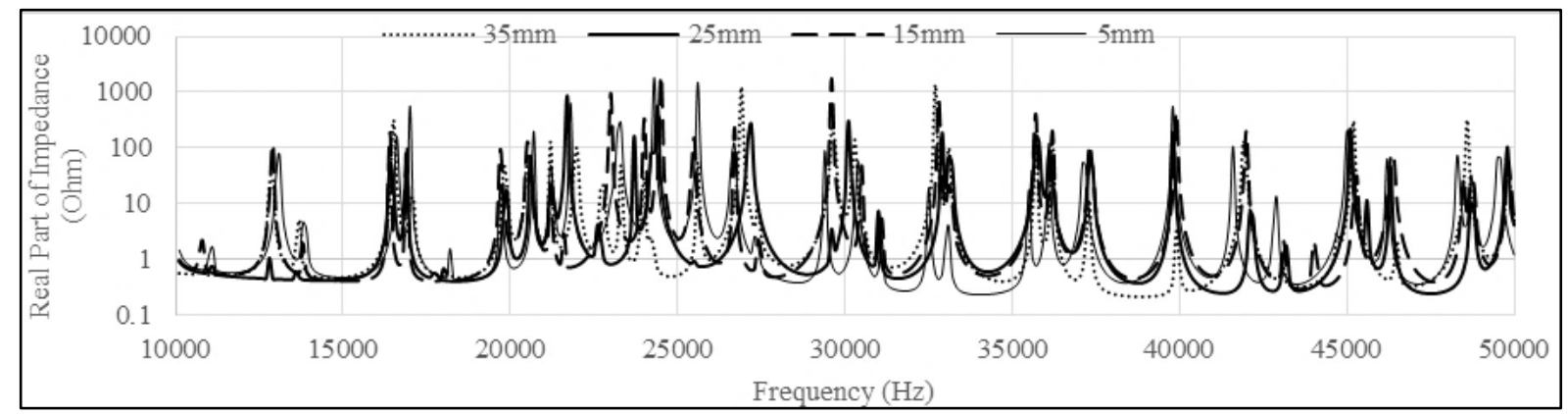

Figure 13: Effect of piezoelectric patch location from the $4 \mathrm{~mm}$ notch.

\subsection{Modelling the effect of load}

After investigating the effect of notch damage, the effect of another type of damage, which is load, is worth investigating. Load is one of the problems occurring to moving components like rotating shafts of turbines. When small metal particles get attached to the main rotating shaft of these sensitive structures, the changes of the modes of vibration or other negative effects on the working principles can seriously damage the total system. Fig. 14 depicts the load position's effect on the impedance signal. The model having a $2 \mathrm{~mm}^{3}$ volume connected to a different position from the beginning of the beam (horizontally) and $0.0065 \mathrm{~m}$ from the vertical edge of the beam. Between the ranges of $20-36 \mathrm{kHz}$, higher amplitudes were generated. The resonance frequencies of the system were not changed. For all three cases, the global 
stiffness and mass were the same. It was found that the peaks for $55 \mathrm{~mm}$ load position seemed to be the highest between $20 \mathrm{kHz}$ to $36 \mathrm{kHz}$. This requires further investigation.

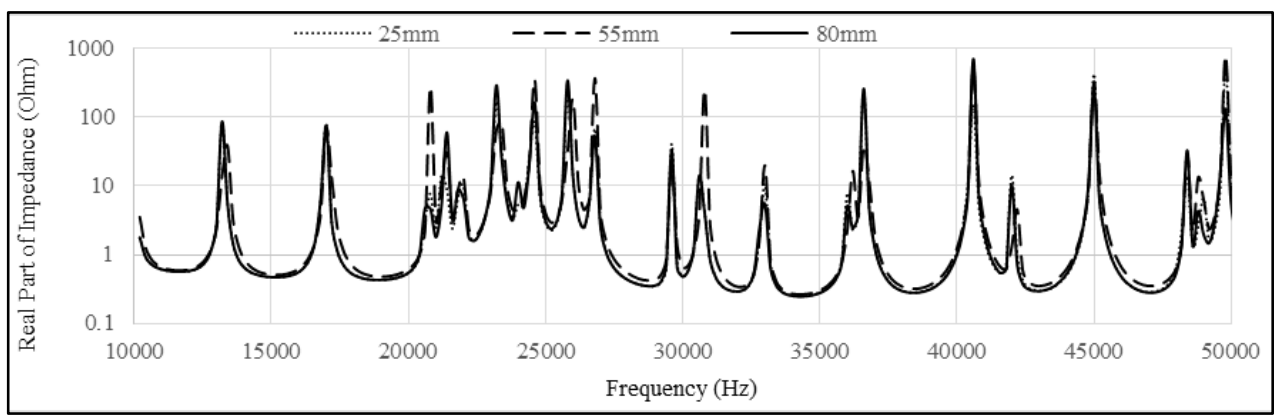

Figure 14: Effect of the location of load from the patch on real part of impedance.

\subsection{Modelling the co-existence of damage and load}

In this part, combining load and crack damage at the same time was attempted in order to model more realistic cases and to prove that EMI systems can discover these problems at the same time. Fig. 15 shows the FE model having load and cracking. Fig. 16 depicts the different signals generated by the load that exist on the notched beam and the healthy beam. New peaks exist for the loaded beam with the $4 \mathrm{~mm}$ notch. It changes the mode and a new resonance is produced. For the notched beam, the global stiffness was reduced, resulting in such resonance generated. However, the presence of the load for the healthy beam and the notched beam did not affect the shifting of the resonance frequency very much. The presence of load alone only produced two higher amplitudes at $21 \mathrm{kHz}$, at $26 \mathrm{kHz}$ and at around $41 \mathrm{kHz}$.

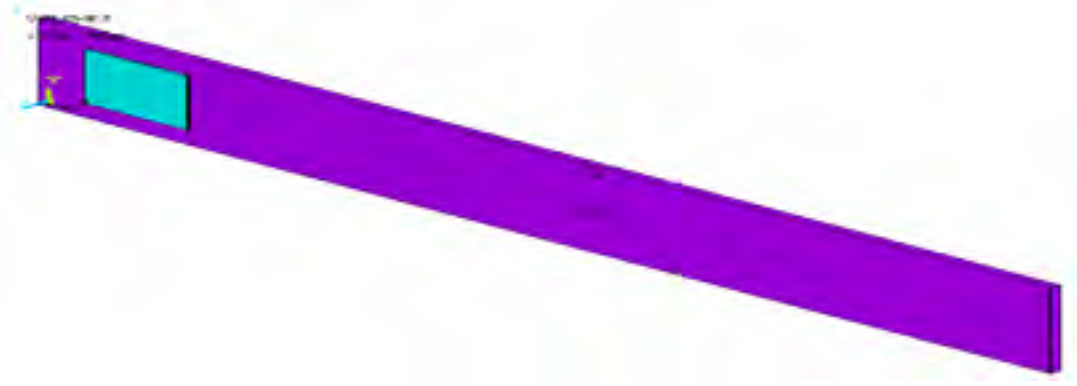

Figure 15: FE model having load and crack.

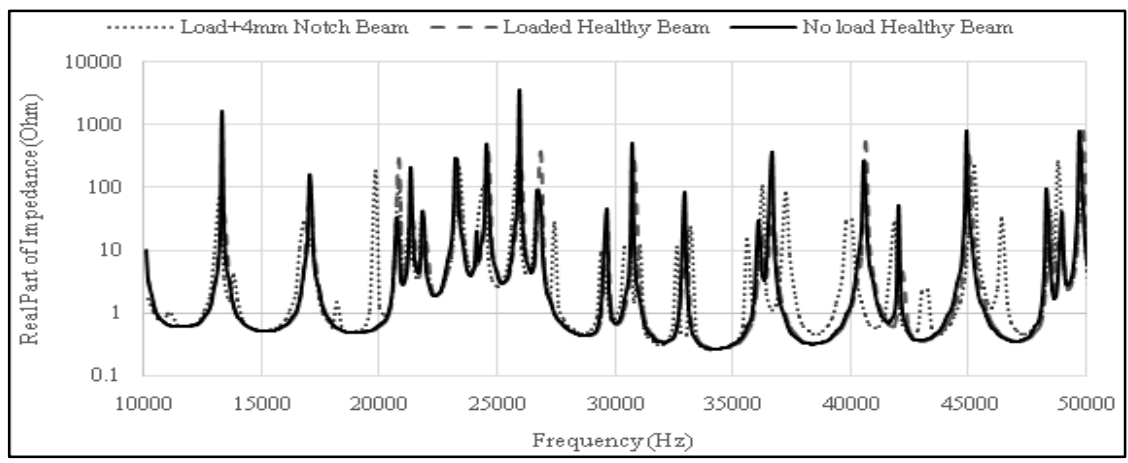

Figure 16: Effect of the coexistence of load and crack the impedance real part.

\section{MODELLING TEMPERATURE EFFECTS ON EMI}

In this section, FE analysis is performed deterministically. The object of examination is an aluminium (alloy 2024) beam in free suspension, with PZT transducers bonded to its surface 
by a thin layer of epoxy. This adhesive is factored into the current model. The beam considered is $500 \mathrm{~mm} \times 30 \mathrm{~mm} \times 2 \mathrm{~mm}$. The PZT measures $15 \mathrm{~mm} \times 15 \mathrm{~mm} \times 0.267 \mathrm{~mm}$. It is made of PSI-5H4E and located $20 \mathrm{~mm}$ from the end of the beam on its left side. 20-node parabolic SOLID226 element was used to build the structure and the PZT models. The simulation was taken a step further, with the inclusion of a temperature effect to the FEM dynamic simulation. It is known that temperature variation can not only change certain host structure properties, but can also affect the PZT itself and its bonding layer. The complete EMI system, which is PZT bonded to the structure with temperature, is simulated. To assess the accuracy of the proposed finite element model, it was subjected to a validation test benchmarked from the literature [21] as shown in Fig. 17. It can be seen that the results show a good agreement and similar resonant frequencies are observed. However, shifting has occurred for most of the peaks. These shifts are due to the lack of the exact experimental data, so that the missing values were assumed.

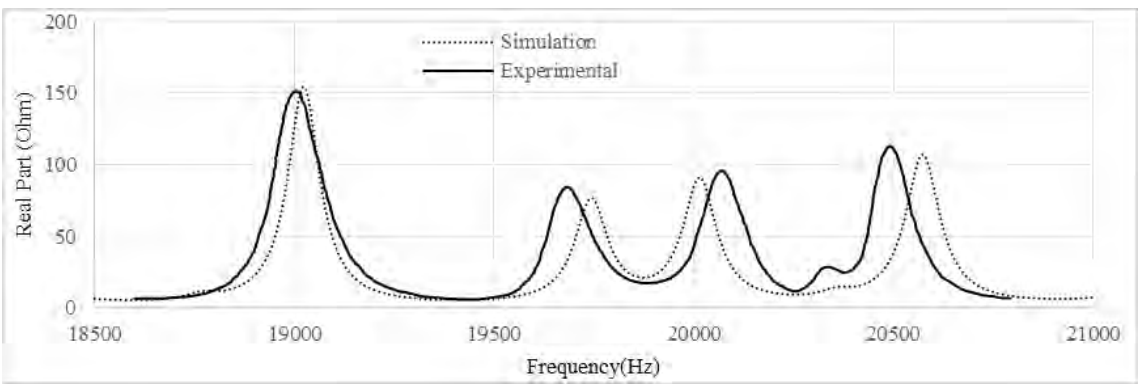

Figure 17: Comparison between simulation and experimental results for an aluminium beam $\left(25^{\circ} \mathrm{C}\right)$.

For FE analysis, the variance of temperature, as it relates to material constants for PSI5H4E PZT patch and 2024 aluminium alloy, are expressed by Eqs. (8) to (9) and Eqs. (10) to (11), respectively, as per Sepehry et al. [22].

$$
\begin{gathered}
\rho=-0.0726 T+7751.8 \\
S_{11}^{E}=-5.5633 \times 10^{-15} T+1.51 \times 10^{-11} \\
\rho=-0.1844 T+2704.6 \\
E=-2.62 T+71.295 \times 10^{9}
\end{gathered}
$$

Simulations were performed at temperatures between 25 and $85^{\circ} \mathrm{C}$. Fig. 18 shows the real part of the electromechanical impedance signatures in a healthy beam for frequencies from 18.5 to $21 \mathrm{kHz}$. As can be seen in Fig. 18, there is a frequency shift to the left for the electromechanical signature due to the increase in temperature. Similarly, as the temperature decreases, the signature shifts to the right. Variations in the electrical resistance and some vertical shifts are also observed.

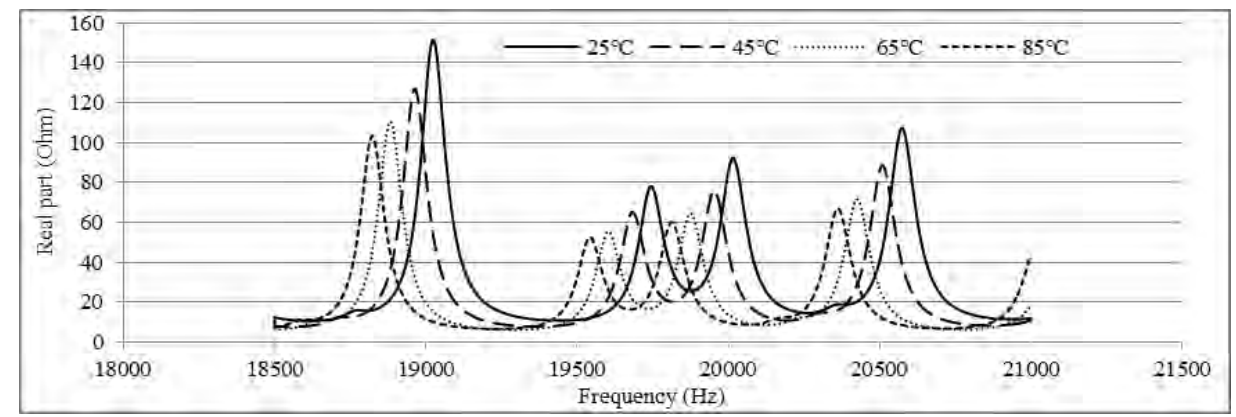

Figure 18: Real part of the electromechanical impedances resulting from temperature changes. 


\section{CONCLUSION}

This paper presented the feasibility of numerical simulation on damage diagnosis using electro-mechanical impedance sensors for monitoring the health of mechanical structures. The effects of different EMI parameters, such as patch geometry (shape and thickness) and bonding layer thickness, were investigated. Then, the temperature effect on the FEM dynamic simulation is observed. The experimental and simulated results showed a close match. The results prove that this system is suitable for use in continuous structure monitoring using coupled field elements, and that direct simulation of electro-mechanical interaction between the host structure and the PZT patch was achievable. This finite element model also successfully verified some vital experimental observations in the literature. This study proves that the FEM could emerge as an excellent alternative to experimentation in the study of the EMI technique. There remains a need for continued numerical and experimental work that would identify, locate and investigate how various types of introduced damage will affect the system.

\section{ACKNOWLEDGMENT}

This work was supported by the International Islamic University Malaysia Endowment Fund [grant number EDW B13-082-0967]. The authors would also like to thank Mrs. Lynn Mason for her assistance in editing this manuscript.

\section{REFERENCES}

[1] Lim, Y. Y.; Soh, C. K. (2012). Effect of varying axial load under fixed boundary condition on admittance signatures of electromechanical impedance technique, Journal of Intelligent Material Systems and Structures, Vol. 23, No. 7, 815-826, doi:10.1177/1045389X12437888

[2] Hamzeloo, S. R.; Shamshirsaz, M.; Rezaei, S. M. (2012). Damage detection on hollow cylinders by electro-mechanical impedance method: Experiments and finite element modeling, Comptes Rendus Mécanique, Vol. 340, No. 9, 668-677, doi:10.1016/j.crme.2012.07.001

[3] Moslemi, M.; Khoshravan, M. (2015). Cohesive zone parameters selection for mode-I prediction of interfacial delamination, Strojniski vestnik - Journal of Mechanical Engineering, Vol. 61, No. 9, 507-516, doi:10.5545/sv-jme.2015.2521

[4] Azhdary, F.; Shabakhty, N. (2014). Performance based design and damages estimation of steel frames with consideration of uncertainties, Technical Gazette, Vol. 21, No. 2, 351-358

[5] Cherrier, O.; Selva, P.; Pommier-Budinger, V.; Lachaud, F.; Morlier, J. (2013). Damage localization map using electromechanical impedance spectrums and inverse distance weighting interpolation: Experimental validation on thin composite structures, Structural Health Monitoring, Vol. 12, No. 4, 311-324, doi:10.1177/1475921713493343

[6] Cortez, E. N.; Vieira Filho, J.; Baptista, F. G. (2013). A new microcontrolled structural health monitoring system based on the electromechanical impedance principle, Structural Health Monitoring, Vol. 12, No. 1, 14-22, doi:10.1177/1475921712461163

[7] Baptista, F. G.; Budoya, D. E.; de Almeida, V. A. D.; Ulson, J. A. C. (2014). An experimental study on the effect of temperature on piezoelectric sensors for impedance-based structural health monitoring, Sensors, Vol. 14, No. 1, 1208-1227, doi:10.3390/s140101208

[8] Annamdas, V. G. M.; Yang, Y.-W.; Soh, C. K. (2007). Influence of loading on electromechanical admittance of piezoceramic transducers, Journal of Smart Materials and Structures, Vol. 16, No. 5, 1888-1897, doi:10.1088/0964-1726/16/5/045

[9] Yan, W.; Cai, J. B.; Chen, W. Q. (2011). An electro-mechanical impedance model of a cracked composite beam with adhesively bonded piezoelectric patches, Journal of Sound and Vibration, Vol. 330, No. 2, 287-307, doi:10.1016/j.jsv.2010.08.013

[10] Liang, C.; Sun, F. P.; Rogers, C. A. (1994). Coupled electro-mechanical analysis of adaptive material systems - determination of the actuator power consumption and system energy transfer, 
Journal of Intelligent Material Systems and Structures, Vol. 5, No. 1, 12-20, doi: $10.1177 / 1045389$ X9400500102

[11] Liu, W.; Giurgiutiu, V. (2007). Finite element simulation of piezoelectric wafer active sensors for structural health monitoring with coupled-filed elements, Proceedings SPIE 6529, Sensors and smart structures technologies for civil, mechanical and aerospace systems 2007, 13 pages, doi: $10.1117 / 12.715238$

[12] Gresil, M., Yu, L.; Giurgiutiu, V.; Sutton, M. (2012). Predictive modeling of electromechanical impedance spectroscopy for composite materials, Journal of Structural Health Monitoring, Vol. 11, No. 6, 671-683, doi:10.1177/1475921712451954

[13] Dare de Almeida, V. A.; Baptista, F. G.; de Aguiar, P. R. (2015). Piezoelectric transducers assessed by the pencil lead break for impedance-based structural health monitoring, IEEE Sensors Journal, Vol. 15, No. 2, doi:10.1109/JSEN.2014.2352171

[14] Rosiek, M.; Martowicz, A.; Uhl, T. (2010). Uncertainty and sensitivity analysis of electromechanical impedance based SHM system, IOP Conference Series: Materials Science and Engineering, Vol. 10, No. 1, 1-9, doi:10.1088/1757-899X/10/1/012207

[15] Dugnani, R. (2009). Dynamic behavior of structure-mounted disk-shape piezoelectric sensors including the adhesive layer, Journal of Intelligent Material Systems and Structures, Vol. 20, No. 13, 1553-1564, doi:10.1177/1045389X08101633

[16] Islam, M. M.; Huang, H. (2014). Understanding the effects of adhesive layer on the electromechanical impedance (EMI) of bonded piezoelectric wafer transducer, Smart Materials and Structures, Vol. 23, No. 12, Paper 125037, doi:10.1088/0964-1726/23/12/125037

[17] Baptista, F. G.; Filho, J. V.; Inman, D. J. (2011). Sizing PZT transducers in impedance-based structural health monitoring, IEEE Sensors Journal, Vol. 11, No. 6, 1405-1414, doi:10.1109/ JSEN.2010.2098865

[18] Bhalla, S.; Yang, Y. W.; Annamdas, V. G. M.; Lim, Y. Y.; Soh, C. K. (2012). Impedance models for structural health monitoring using piezo-impedance transducers, Soh, C. K.; Yang, Y.; Bhalla, S. (Eds.), Smart Materials in Structural Health Monitoring, Control and Biomechanics, SpringerVerlag, Berlin, 53-128, doi:10.1007/978-3-642-24463-6 3

[19] Ong, C. W.; Yang, Y.; Wong, Y. T.; Bhalla, S.; Lu, Y.; Soh, C. K. (2003). Effects of adhesive on the electromechanical response of a piezoceramic-transducer-coupled smart system, Proceedings SPIE 5062, Smart Materials, Structures, and Systems, 241-247, doi:10.1117/12.514757

[20] Yang, Y.; Lim, Y. Y.; Soh, C. K. (2008). Practical issues related to the application of the electromechanical impedance technique in the structural health monitoring of civil structures: I. Experiment, Smart Materials and Structures, Vol. 17, No. 3, Paper 035008, doi:10.1088/0964$1726 / 17 / 3 / 035008$

[21] Baptista, F. G.; Filho, J. V.; Inman, D. J. (2012). Real-time multi-sensors measurement system with temperature effects compensation for impedance-based structural health monitoring, Structural Health Monitoring, Vol. 11, No. 2, 173-186, doi:10.1177/1475921711414234

[22] Sepehry, N.; Shamshirsaz, M.; Bastani, A. (2011). Experimental and theoretical analysis in impedance-based structural health monitoring with varying temperature, Structural Health Monitoring, Vol. 10, No. 6, 573-585, doi:10.1177/1475921710388338 